\title{
Compression of the Medulla Oblongata and Acute Respiratory Failure Caused by Rupture of a Thrombosed Large Aneurysm of the Anterior Inferior Cerebellar Artery
} -Case Report-

\author{
Hirofumi OYAMA, Akira KITO, Hideki MAKI, \\ Kenichi HATTORI, and Kuniaki TANAHASHI
}

Department of Neurosurgery, Ogaki Municipal Hospital, Ogaki, Gifu

\begin{abstract}
A 65-year-old female presented with an extremely rare case of a ruptured thrombosed large aneurysm of the anterior inferior cerebellar artery (AICA) in which a local hematoma compressed the medulla oblongata and caused acute respiratory failure. She first presented with dizziness, general fatigue, and nausea 2 months before admission. She was hospitalized for intense general fatigue, nausea, and occipitalgia. Computed tomography and $T_{1}$-weighted magnetic resonance imaging showed a heterogeneous lesion around the right cerebello-medullary cistern. Angiography revealed a fusiform aneurysm of the right AICA. Asphyxia occurred 4 days after admission and the patient underwent an emergency operation. No subarachnoid hematoma was present, but a hematoma around the ruptured portion markedly compressed the medulla oblongata. The medulla oblongata was adequately decompressed after subtotal removal of the aneurysm. The patient's respiratory status and consciousness level recovered after the operation.
\end{abstract}

Key words: thrombosed aneurysm, anterior inferior cerebellar artery, medulla oblongata, occipital artery-anterior inferior cerebellar artery anastomosis, respiratory failure

\section{Introduction}

Aneurysm of the anterior inferior cerebellar artery (AICA) is relatively rare, and generally occurs as saccular or dissecting aneurysm. ${ }^{2-5,8-12,14,15,17)}$ Progressive growth of an aneurysm of the AICA generally manifests as various cerebellopontine angle syndromes such as oculomotor nerve palsy, trigeminal sensory loss or neuralgia, sixth cranial nerve palsy, seventh cranial nerve palsy, vertigo, tinnitus, hearing loss, lower cranial nerve palsy, and ataxia. $1,6,7,10,11,13,14,16)$ Rapid deterioration of neurological symptoms generally indicates rupture of an aneurysm. Subarachnoid hemorrhage may subsequently cause dyspnea, arrhythmia, and death after these cerebellopontine angle syndromes occur.9) However, only subarachnoid hemorrhage does not cause rapid neurological deterioration.

We report an extremely rare case of a ruptured thrombosed large aneurysm in which a local hematoma, not a diffuse subarachnoid hematoma, compressed the medulla oblongata and caused acute respiratory failure.

Received July 23, 2009;

Accepted December 11, 2009

\section{Case Report}

A 65-year-old female suffered dizziness, general fatigue, and nausea 2 months before admission. She was hospitalized for intense general fatigue, nausea, and occipitalgia. On admission, she complained of paresthesia throughout her whole body, diplopia, and oscillopsia. Neurological examination revealed dysmetria in the bilateral upper extremities, dysarthria, and dysesthesia in the left limbs.

Computed tomography (CT) revealed a heterogeneous high density mass lesion around the right cerebello-medullary cistern (Fig. 1A). CT with contrast medium showed that the non-thrombosed lumen was surrounded by the thrombosed portion (Fig. 1B). $\mathrm{T}_{1}$-weighted magnetic resonance (MR) imaging showed a slightly hyperintense lesion around the right cerebello-medullary cistern (Fig. 1C). MR imaging with contrast medium demonstrated that the non-thrombosed lumen was surrounded by the heterogeneous mass lesion (Fig. 1D). Vertebral angiography revealed a fusiform aneurysm in the pre-meatal segment of the right AICA (Fig. 2). We decided to treat this aneurysm surgically in the subacute stage.

The patient suffered sudden onset of respiratory arrest which required intubation and artificial respiration 4 days 


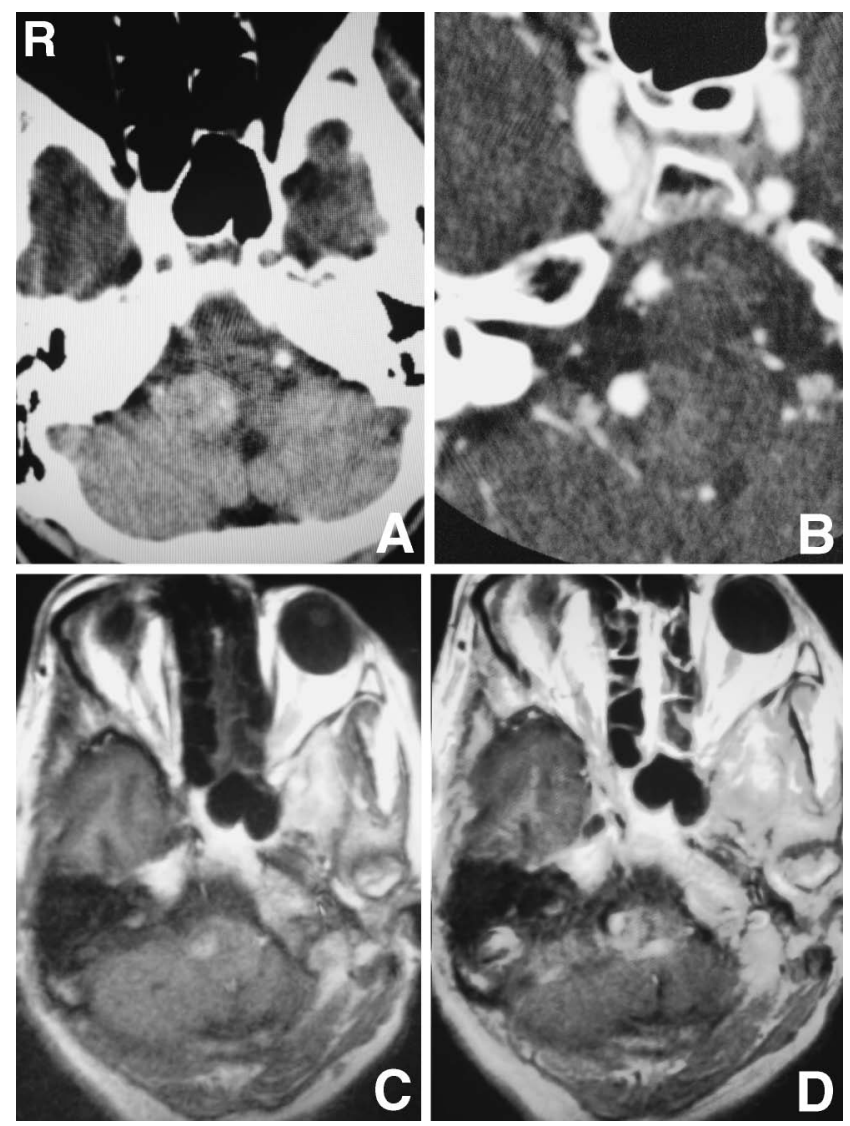

Fig. 1 A: Preoperative computed tomography scan showing a heterogeneous high-density mass lesion around the right cerebello-medullary cistern. B: Preoperative computed tomography scan with contrast medium showing the nonthrombosed lumen surrounded by the thrombosed portion. C: Preoperative $T_{1}$-weighted magnetic resonance image showing a slightly hyperintense lesion around the right cerebello-medullary cistern. $D$ : Preoperative $T_{1}$-weighted magnetic resonance image with gadolinium showing the non-thrombosed lumen surrounded by the heterogeneous mass lesion.

after admission. Spontaneous respiration resumed after tracheal aspiration, and she became able to nod her head. Emergency suboccipital retromastoid craniotomy was performed. Exploration of the posterior fossa disclosed a large, partially thrombosed, fusiform aneurysm of the AICA, located just anterior to the cranial nerves VII, VIII, IX, X, and XI, and cephalad to the cranial nerve XII (Fig. 3A). No subarachnoid hematoma was present. The occipital artery was first anastomosed to the hemispheric portion of the right AICA. The aneurysm was trapped and partially removed, except for the portion adhered to the brain stem. The caudal one-third of the aneurysm had a very fragile outer wall (Fig. 3A). After dissecting the caudal wall from the medulla oblongata, fresh clot was observed between this wall and the medulla oblongata (Fig. 3B). The fresh clot had markedly compressed the medulla oblongata (Fig. 3C). After thrombectomy of the rostral two-thirds of the aneurysm, a partially thrombosed clot was observed at the bottom of the aneurysm wall. This was

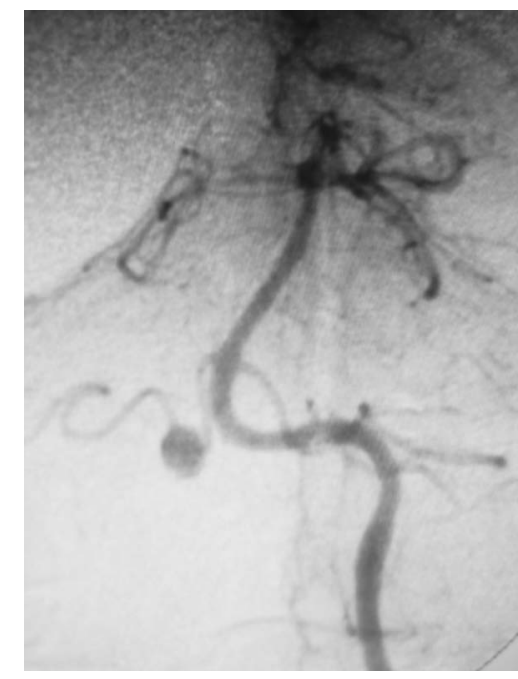

Fig. 2 Left vertebral angiogram showing a fusiform aneurysm in the pre-meatal segment of the right anterior inferior cerebellar artery.
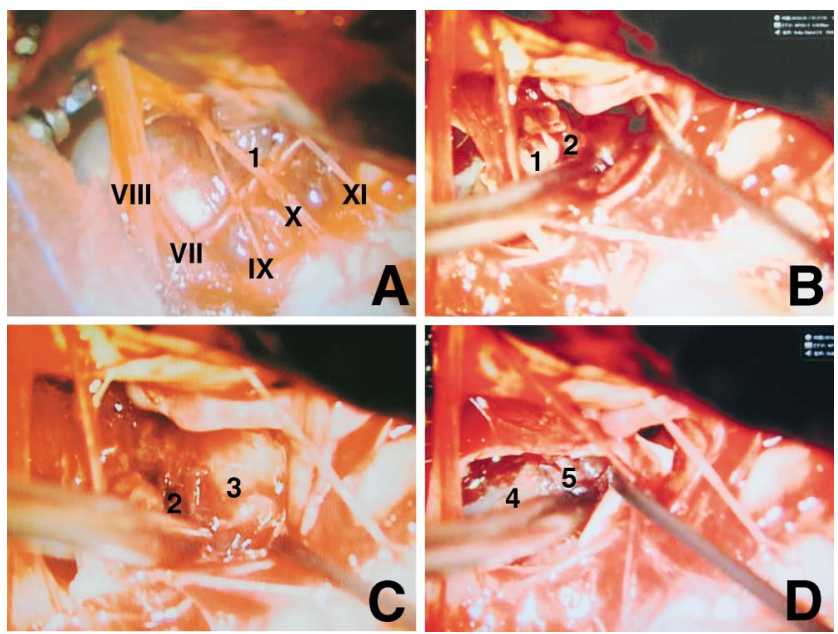

Fig. 3 A: Intraoperative microscope view before thrombectomy of posterior fossa exploration revealing a large, partially thrombosed, fusiform anterior inferior cerebellar artery aneurysm located just anterior to the cranial nerves VII, VIII, IX, X, and XI. The caudal one-third of the aneurysm has a very fragile outer wall (1). B: Intraoperative microscope view after dissection of the caudal wall from the medulla oblongata showing fresh clot (2) between the caudal wall (1) and the medulla oblongata. C: Intraoperative microscope view after removal of the hematoma around the medulla oblongata showing fresh clot (2) markedly compressing the medulla oblongata (3). D: Intraoperative microscope view after thrombectomy of the rostral two-thirds of the aneurysm showing the wall of the aneurysm (4) remains adhered to brain stem, and partially thrombosed clot (5) at the bottom of the aneurysm cavity indicating the suspected site of rupture.

the suspected location of the rupture (Fig. 3D). The medulla oblongata was adequately decompressed after subtotal 
removal of the aneurysm.

After the operation, the patient developed methicillinresistant Staphylococcus aureus meningitis due to cerebrospinal fluid leakage through the scalp crust. The infected subgaleal space was drained and systemic administration of vancomycin was begun, but caused Stevens-Johnson syndrome. However, the patient recovered with plasma exchange therapy. She needed a tracheostomy for excessive phlegm production and remained wheelchair-dependent for a while. She had dysphagia, left hemiplegia, and gait disturbance. However, her sensorium improved to normal. She later underwent rehabilitation for walking and writing.

\section{Discussion}

In the present case, the outer wall of the aneurysm was very fragile locally around the medulla oblongata and a fresh clot markedly compressed the brain stem. These findings suggested rupture, despite the absence of subarachnoid hematoma. Enlargement of an aneurysm may cause gradual neurological decline, but the abrupt onset of respiratory arrest in our patient indicated that the brain stem was drastically compressed by the clot around the aneurysm.

A few cases of thrombosed large AICA aneurysm have been reported, ${ }^{1,6,7,11,13,16)}$ including local association with acute hematoma. ${ }^{6,16)}$ One patient complained of sudden onset of occipital headache, nausea, and vomiting, ${ }^{6)}$ and the other patient suddenly developed facial numbness and headache. ${ }^{16)}$ The sudden onsets of symptoms indicated rupture of an aneurysm in these cases. However, only the hematoma around the aneurysm was detected. Our patient required urgent hospitalization for intense general fatigue, nausea, and occipitalgia. The hemorrhage may already have occurred at that time, but the hematoma outside the aneurysm was very difficult to discriminate from the thrombosis inside the aneurysm on CT and MR imaging.

Endovascular obliteration of the AICA and the aneurysm is one possible treatment. , $^{2,3,8,8,9,12,17)}$ Coil embolization of the aneurysm dome was potentially very risky in our patient because the outer aneurysm wall was locally very fragile. We selected direct surgery to decompress the brain stem. The AICA needs to be reconstructed after trapping or resection. Occlusion of this artery of parapontine area without revascularization may carry the risk of death, hearing loss, and/or cerebellar infarction. ${ }^{4}$ In our patient, the perforating artery was not included in the trapping range, and the distal portion of the AICA was vascularized by occipital artery-AICA anastomosis. Therefore, the risk of ischemic event could be avoided after trapping.

\section{References}

1) Cantore GP, Ciappetta P, Vagnozzi R, Bozzao L: Giant aneurysm of the anterior inferior cerebellar artery simulating a cerebellopontine angle tumor. Surg Neurol 18: 76-78, 1982

2) Choi $\mathrm{CH}$, Cho WH, Choi BK, Lee SW: Rerupture following endovascular treatment for dissecting aneurysm of distal anterior inferior cerebellar artery with parent artery preservation: retreatment by parent artery occlusion with Guglielmi detachable coils. Acta Neurochir (Wien) 148: 363-366, 2006

3) Fukushima S, Hirohata M, Okamoto Y, Yamashita S, Ishida S, Shigemori M: Anterior inferior cerebellar artery dissecting aneurysm in a juvenile: case report. Neurol Med Chir (Tokyo) 49: 81-84, 2009

4) Gi H, Inoha S, Uno J, Ikai Y, Koga H, Yamaguchi S, Nagaoka S: [Four cases of direct surgery for anterior inferior cerebellar artery aneurysms]. No Shinkei Geka 35: 571-578, 2007 (Japanese)

5) Hancock JH, Millar JS: Spontaneous dissection of the anterior inferior cerebellar artery. Neuroradiology 42: 535-538, 2000

6) Ildan F, Göçer AI, Bağdatoğlu H, Uzuneyüpoğlu Z, Tuna M, Cetinalp E: Isolated trigeminal neuralgia secondary to distal anterior inferior cerebellar artery aneurysm. Neurosurg Rev 19: 43-46, 1996

7) Johnson JH Jr, Kline DG: Anterior inferior cerebellar artery aneurysms. Case report. J Neurosurg 48: 455-460, 1978

8) Kusaka N, Maruo T, Nishiguchi M, Takayama K, Maeda Y, Ogihara K, Nakagawa M, Gotoh M, Nishiura T: [Embolization for aneurismal dilatation associated with ruptured dissecting anterior inferior cerebellar artery aneurysm with preservation of the parent artery: case report]. No Shinkei Geka 34: 729-734, 2006 (Japanese)

9) Matsuyama T, Okuchi K, Norimoto K, Ueyama T: Ruptured dissecting anterior inferior cerebellar artery aneurysm-case report. Neurol Med Chir (Tokyo) 42: 214-216, 2002

10) Nishimoto A, Fujimoto $S$, Tsuchimoto $S$, Matsumoto $Y$, Tabuchi K, Higashi T: Anterior inferior cerebellar artery aneurysm. Report of three cases. J Neurosurg 59: 697-702, 1983

11) Pritz MB: Aneurysms of the anterior inferior cerebellar artery. Acta Neurochir (Wien) 120: 12-19, 1993

12) Saito A, Ezura M, Takahashi A, Yoshimoto T: [An arterial dissection of the distal anterior inferior cerebellar artery treated by endovascular therapy]. No Shinkei Geka 28: 269-274, 2000 (Japanese)

13) Sarkar A, Link MJ: Distal anterior inferior cerebellar artery aneurysm masquerading as a cerebellopontine angle tumor: case report and review of literature. Skull Base 14: 101-106, 2004

14) Schwartz HG: Arterial aneurysm of the posterior fossa. J Neurosurg 5: 312-316, 1948

15) Takeuchi S, Takasato $Y$, Masaoka H, Hayakawa T, Otani N, Yoshino Y, Yatsushige H: [Trapping of ruptured dissecting aneurysm of distal anterior inferior cerebellar artery-case report]. Brain Nerve 61: 203-207, 2009 (Japanese)

16) Zager EL: Isolated trigeminal sensory loss secondary to a distal anterior inferior cerebellar artery aneurysm: case report. Neurosurgery 28: 288-291, 1991

17) Zager EL, Shaver EG, Hurst RW, Flamm ES: Distal anterior inferior cerebellar artery aneurysms. Report of four cases. J Neurosurg 97: 692-696, 2002

Address reprint requests to: Hirofumi Oyama, M.D., Department of Neurosurgery, Ogaki Municipal Hospital, 4-86 Minamikawa-machi, Ogaki, Gifu 503-8502, Japan. e-mail: oya3776@arrow.ocn.ne.jp 